\title{
POSITIVE SUPERHARMONIC FUNCTIONS AND THE HÖLDER CONTINUITY OF CONFORMAL MAPPINGS
}

\author{
J. M. ANDERSON AND A. HINKKANEN
}

\begin{abstract}
We study the rate at which a positive superharmonic function $u$ can tend to zero at a boundary point $z_{0}$ of a plane domain $G$. In particular, if $G$ is a quasidisk, and $\alpha>0$ is given, we show that the condition that $\lim \inf u(z) / \operatorname{dist}(z, \partial G)^{1 / \alpha}>0$ as $z \rightarrow z_{0}$ in $G$ for any such $u$ is related to the condition that the conformal map $f$ of the unit disk onto $G$ with $f(1)=z_{0}$ is Hölder continuous with exponent $\alpha$ at the point 1 . This leads us to consider the problem of finding the best exponent $\alpha$ for which $f$ is Hölder continuous. The answer depends on how we characterize quasidisks or quasicircles. In this connection we give a negative answer to a question of Näkki and Palka.
\end{abstract}

\section{Introduction}

1.1. Let $G$ denote a plane domain and suppose that $u$ is a positive superharmonic function in $G$. We started our investigation by considering the question, suggested by the results in $[7,8]$, of at what rate $u(z)$ can tend to zero as $z$ approaches a point on the boundary $\partial G$ of $G$, at least if $\partial G$ satisfies some regularity conditions. Our conclusions, whose proof is rather elementary, lead us to consider the more difficult question concerning the best exponent of Hölder continuity of a conformal mapping of the unit disk onto a quasidisk $D$ when the quasicircle $\partial D$ is parametrized by means of cross ratios. In that connection, our main result is Theorem 4, stated in Section 3 below, which disproves a conjecture of Näkki and Palka [11] concerning the order of magnitude of that best exponent.

We start by explaining our results for positive superharmonic functions $u$ in a domain $G$. It is well known that if $G$ is the unit disk $\Delta=\{z:|z|<1\}$ then

$$
\liminf _{|z| \rightarrow 1^{-}} u(z)(1-|z|)^{-1}>0
$$

and a nuniber of recent results (see, for example, [7] or [8]) have extended this result to other classes of domains. For a set $E$, we let $d(z, E)$ denote the distance from z to the set $E$. Recently, Kuran has shown, for a class of domains $G$ known as $a$-admissible (see [7, p. 269] for the definition) that

$$
\liminf _{z \rightarrow i G} u(z)(d(z, \partial G))^{-1}>1 \text {. }
$$

Moreover, there is a local version of this result [7, Theorem 2, p. 269] in that if $G$ is merely $a$-admissible at a point $z_{0} \in \partial G$ then

$$
\liminf _{\substack{z \rightarrow z_{0} \\ z \in G}} u(z)(d(z, \partial G))^{-1}>0 .
$$

Received 11 April 1988.

1980 Mathematics Subject Classification (1985 Revision) $30 \mathrm{C60}$.

Research of the second author was partially supported by the US National Science Foundation. 
It will turn out that an important consideration in such results is the behaviour of the conformal mapping from $\Delta$ to a neighbourhood of a point $z_{0} \in \partial G$. It is convenient to formulate the following elementary result as a theorem. We denote the extended complex plane by $\overline{\mathbb{C}}$.

THEOREM 1. Let $G$ be a domain in $\overline{\mathbb{C}}$ with $z_{0}$ a finite boundary point of $G$. Suppose that there is a Jordan curve or a non-degenerate Jordan arc $\Gamma$ and a neighbourhood $U$ of $z_{0}$ such that $U \cap \partial G$ is an arc of $\Gamma$. Let $D$ be a component of $\overline{\mathbb{C}} \backslash \Gamma$ for which $D \cap G \cap V$ is not empty for any neighbourhood $V$ of $z_{0}$, and let $f$ be a conformal mapping of $\Delta$ onto $D$ with $f(1)=z_{0}$. Suppose that there are positive constants $M, \varepsilon$ and $\alpha$ such that

$$
\left|f^{\prime}(z)\right| \leqslant M(1-|z|)^{\alpha-1}
$$

for $|z|<1,|z-1|<\varepsilon$. Then, for any positive superharmonic function $u$ defined in $G$ we have

$$
\liminf _{\substack{z \rightarrow z_{0} \\ z \in G \cap D}} u(z)(d(z, \partial G))^{-1 / \alpha}>0 .
$$

The open set $\overline{\mathbb{C}} \backslash \Gamma$ has one or two components, and if there are two of them, say $D_{1}$ and $D_{2}$, then $G \cap D_{i} \cap V$ might be non-empty for both $i=1$ and $i=2$, for any neighbourhood $V$ of $z_{0}$. If so, then we have (1.3) as $z \rightarrow z_{0}$ in $G$ provided that the conformal mapping $f_{i}$ of $\Delta$ onto $D_{i}$, with $f_{i}(1)=z_{0}$, satisfies (1.2) (with $f$ replaced by $f_{i}$ ) for $i=1,2$.

If, for example, $\partial G$ consists of countably many disjoint Jordan arcs and curves that cluster at most at infinity, then each finite $z_{0} \in \partial G$ has a neighbourhood $U$ as in Theorem 1 (it is, however, another matter to determine under what circumstances (1.2) holds).

We shall be concerned with the case $0<\alpha \leqslant 1$. For if $\alpha>1$, then (1.2) implies that $f^{\prime}(z) \rightarrow 0$ as $z$ tends to any point on an arc of $\partial \Delta$. By Privalov's theorem, $f^{\prime} \equiv 0$ so that $f$ is constant and hence not conformal. If $0<\alpha \leqslant 1$ and if (1.2) holds for all $z \in \Delta$ then a well-known result of Hardy and Littlewood [6] asserts that $f \in \operatorname{Lip}(\alpha, \bar{\Delta})$ and conversely (see, for example, [5, Theorem 5.1]). We say, of course, that $f \in \operatorname{Lip}(\alpha, \bar{\Delta})$ if and only if

$$
\left|f\left(z_{1}\right)-f\left(z_{2}\right)\right| \leqslant M_{1}\left|z_{1}-z_{2}\right|^{\alpha}
$$

for some positive $M_{1}$ and all $z_{1}, z_{2} \in \bar{\Delta}$.

1.2. To prove Theorem 1 , let $g$ be a conformal map of $\Delta$ onto

$$
V_{1}=\left\{z:|z-1|<\varepsilon_{1}\right\} \cap \Delta
$$

with $g(1)=1$ where $\varepsilon_{1}$ is so small that $\varepsilon_{1}<\varepsilon$ and $f\left(V_{1}\right) \subset G$. By reflection, we extend $g$ to a conformal map of $\Delta \cup\{z:|z-1|<\delta\}$ onto some neighbourhood of the point.l. We take $\delta \in\left(0, \varepsilon_{1}\right)$ so small that $1 / c \leqslant\left|g^{\prime}(z)\right| \leqslant c$ for some $c>1$ whenever $|z-1|<\delta / 2$ and thus also if $|g(z)-1|<\delta_{1}$, for some $\delta_{1} \in(0, \delta)$.

By [8, Proposition 1, p. 199], the positive superharmonic function $v=u \circ f \circ g$ defined in $\Delta$ satisfies

$$
\liminf _{z \rightarrow 1} v(z)(1-|z|)^{-1}>0 .
$$

Let $\rho_{D}$ denote the Poincaré density in $D$. Since

$$
\left|f^{\prime}(g(z)) g^{\prime}(z)\right|\left(1-|z|^{2}\right)=\rho_{D}((f \circ g)(z))^{-1} \geqslant d((f \circ g)(z), \partial D)
$$


and $d(w, \partial D)=d(w, \partial G)$ when $\left|w-z_{0}\right|$ is small enough, and since

$$
1-|g(z)| \geqslant(1-|z|) / c_{1}
$$

for some $c_{1}>1$ when $|z-1|$ is small enough, we see after some calculations that (1.2) together with the above estimates implies (1.3). This proves Theorem 1.

1.3. The relation of our problem to that of conformal mapping is further illustrated by the following rather simple observation.

THEOREM 2. Let $g$ map the Jordan domain $G$ conformally onto the upper half-plane or onto the semidisk $\{z:|z|<1$ and $\operatorname{Im} z>0\}$ with $g\left(z_{0}\right)=0$ where $z_{0}$ is a finite boundary point of $G$. Then for any positive superharmonic function $u$ in $G$ we have

$$
\liminf _{z \rightarrow z_{0}} \frac{u(z)}{\operatorname{Im} g(z)}>0 .
$$

Since $\operatorname{Im} g$ is a positive harmonic function in $G$, it follows that $\operatorname{Im} g$ gives the slowest rate at which a positive superharmonic function in $G$ can tend to zero as $z \rightarrow z_{0}$.

In Theorem 2, we could assume that $G$ is a general simply connected domain, not necessarily a Jordan domain, provided that $\left\{z_{0}\right\}$ is the impression of a prime end and that the point $z_{0}$ belongs to the impression of only one prime end. Under these assumptions, the statement $g\left(z_{0}\right)=0$ makes sense.

To prove Theorem 2, we note that the result (1.1) for the unit disk can be used together with a conformal mapping argument to deduce that the positive superharmonic function $v=u \circ g^{-1}$ defined in $g(G)$ satisfies

$$
\liminf _{\substack{i \in \rightarrow 0 \\ w \in g(G)}} \frac{v(w)}{\operatorname{Im} w}>0 .
$$

Here $g(G)$ is the upper half-plane or a semidisk. Replacing $w$ by $g(z)$ in (1.6) we obtain (1.5). This proves Theorem 2.

1.4. When $G$ is simply connected, there is a converse relation between (1.2) and (1.3).

THEOREM 3. Suppose that the conformal map $f$ of $\Delta$ onto the simply connected domain $G$ in $\overline{\mathbb{C}}$ with the finite boundary point $z_{0}$ satisfies $f(z) \rightarrow z_{0}$ as $z \rightarrow 1$ in $\Delta$. Suppose that $\alpha>0$ and that

$$
\liminf _{\substack{z \rightarrow z_{0} \\ z \in G}} \frac{u(z)}{d(z, \partial G)^{1 / \alpha}}>0
$$

whenever $u$ is a positive superharmonic function in $G$. Then there are positive numbers $\varepsilon, M$ and $M_{1}$, independent of $u$, such that (1.2) holds for all $z \in W=\Delta \cap\{z:|z-1|<\varepsilon\}$ and such that (1.4) holds for all $z_{1}, z_{2} \in W$.

The conclusion (1.2) shows that we must have had $0<\alpha \leqslant 1$. To prove Theorem 3 , we apply (1.7) to $u(z)=-\log \left|f^{-1}(z)\right|$ and note that $u(z) \leqslant 2\left(1-\left|f^{-1}(z)\right|^{2}\right)$ when $\left|f^{-1}(z)\right| \geqslant \frac{1}{2}$. Let $\rho_{c}$ be the Poincaré density in $G$. Since

$$
d(z, \partial G) \geqslant c \rho_{i}(z)^{-1}=c\left(1-\left|f^{-1}(z)\right|^{2}\right)\left|f^{\prime}\left(f^{-1}(z)\right)\right|
$$


as $z \rightarrow z_{0}$ in $G$, we obtain (1.2), and hence (1.4) by integration (cf. [6, p. 427]). Note that if $\infty \notin G$, then (1.8) holds for all $z \in G$ with $c=\frac{1}{4}$, while if $\infty \in G$, then (1.8) holds with, say, $c=\frac{1}{8}$ when $\left|z-z_{0}\right|$ is small enough. This proves Theorem 3 .

\section{Quasidisks: the standard definition}

Suppose that $G$ is a Jordan domain in $\overline{\mathbb{C}}$, and let $f$ map $\Delta$ conformally onto $G$. If $f$ has a quasiconformal extension $F$ to $\overline{\mathbb{C}}$ with complex dilatation $\mu=F_{z} / F_{z}$ and if further the $L^{\infty}$-norm $\|\mu\|_{x}$ of $\mu$ satisfies $\|\mu\|_{\infty} \leqslant \kappa<1$, then $G$ is called a $\kappa$-quasidisk. Simple calculations together with [12, Lemma 9.9, p. 287] show that then for any $\zeta \in \partial \Delta$ with $F(\zeta) \neq \infty$ there are positive numbers $M$ and $\varepsilon$ such that

$$
\left|f^{\prime}(z)\right| \leqslant M(1-|z|)^{-\kappa}
$$

for $|z|<1,|z-\zeta|<\varepsilon$. Thus (1.2) holds with $\alpha=1-\kappa$, and so Theorem 1 yields the following consequence.

COROllaRY 1. Let $G$ be a domain in $\overline{\mathbb{C}}$ whose boundary consists of finitely many, disjoint quasicircles, and suppose that for each component $\Gamma$ of $\partial G$, the component of $\overline{\mathbb{C}} \backslash \Gamma$ containing $G$ is a $\kappa$-quasidisk. Then for any positive superharmonic function $u$ in $G$ and for each finite $z_{0} \in \partial G$, (1.3) holds with $\alpha=1-\kappa$ as $z \rightarrow z_{0}$ in $G$.

The following example shows that the conclusion of Corollary 1 cannot, in general, be improved. The various assertions that we make about this example will not be proved here since the proofs involve only routine considerations. For a fixed $\kappa$ with $0<\kappa<1$, we set

$$
G_{1}=\{z:|\arg z|<(1-\kappa) \pi / 2\}
$$

and take $G=\phi\left(G_{1}\right)$ where $\phi(z)=(z-1) /(z+1)$. Then $G_{1}$ and $G$ are $\kappa$-quasidisks (cf. [2, pp. 81-82], where $1-\kappa$ is replaced by $1+\kappa$ even though a similar argument works for $1-\kappa$ also). For $\operatorname{Re} z>0$, we define $\psi(z)=z^{1 /(1-\kappa)}$, taking that branch which is positive for $z=x>0$. Now set $g=\phi \circ \psi \circ \phi^{-1}$ so that $g$ maps $G$ conformally onto $\Delta$ and $g(z)$ is real for real $z$.

We choose $z_{0}=-1$ and note that

$$
g(z)+1 \sim 2^{-\kappa /(1-\kappa)}(z+1)^{1 /(1-\kappa)}
$$

as $z \rightarrow-1$ along the real axis in $G$. The function $u(z)=1+\operatorname{Re} g(z)$ is positive and harmonic in $G$. Moreover, as $z \rightarrow-1$ along the real axis is $G$, we have

$$
d(z, \partial G) \sim(z+1) \sin [(1-\kappa) \pi / 2] .
$$

Hence

$$
\lim _{\substack{z \rightarrow-1, z \in G \\ z \text { real }}} \frac{u(z)}{d(z, \partial G)^{1 /\left(1-\kappa^{\prime}\right)}}=\left(2^{\kappa} \sin [(1-\kappa) \pi / 2]\right)^{1 /(\kappa-1)}>0 .
$$

\section{Quasidisks: the geometric definition}

In [1, p. 295] Ahlfors gave a geometric characterization of quasicircles. This led Gehring to formulate the following definition, which seems to have first appeared in [3] and subsequently in [4]. A Jordan curve $\Gamma$ in $\overline{\mathbb{C}}$ is called a $k$-circle where $0<k \leqslant 1$ if and only if

$$
\frac{\left|z_{1}-z_{3}\right|\left|z_{2}-z_{4}\right|}{\left|z_{1}-z_{2}\right|\left|z_{3}-z_{4}\right|+\left|z_{2}-z_{3}\right|\left|z_{4}-z_{1}\right|} \geqslant k
$$


whenever $z_{1}, z_{2}, z_{3}$ and $z_{4}$ are distinct points of $\Gamma$ that follow each other in the positive or negative direction on $\Gamma$. If one of the $z_{i}$ is infinite, we interpret the left-hand side of (3.1) in the obvious way. A curve $\Gamma$ is a 1-circle if and only if $\Gamma$ is a circle or a straight line. We further note that a $k$-circle is the boundary of a $\kappa$-quasidisk where $\kappa$ depends on $k$ only, and that the boundary of any $\kappa$-quasidisk is a $k$-circle for some $k$ depending on $\kappa$ only.

The standard examples of curves which are $k$-circles are

$$
\left.\begin{array}{l}
\Gamma_{1}=\{0, \infty\} \cup\{z:|\arg z|=\arcsin k\}, \\
\Gamma_{2}=\{0, \infty\} \cup\{z:|\arg z|=\pi-\arcsin k\},
\end{array}\right\}
$$

as well as $\phi\left(\Gamma_{1}\right)$ and $\phi\left(\Gamma_{2}\right)$ where $\phi(z)=(z-1) /(z+1)$ as before (see, for example, [3, pp. 7-10]).

Näkki and Palka have shown in [11, Theorem 2, p. 486] that if $f$ maps $\Delta$ conformally onto a bounded domain $D$ whose boundary is a $k$-circle then $f \in \operatorname{Lip}\left(\alpha_{0}, \bar{\Delta}\right)$, where $\alpha_{0}=\alpha_{0}(k)$ is given by

$$
\alpha_{0}=\frac{2(\arcsin k)^{2}}{\pi(\pi-\arcsin k)} .
$$

It follows from Theorem 1 and the remark after it that, for such a domain $D$, the inequality (1.3) holds with $\alpha=\alpha_{0}$. This value of $\alpha_{0}$ arises from an estimate, [11, Lemma 4, p. 492], for the moduli of certain path families, using only weakly the fact that $\partial D$ is a $k$-circle. Näkki and Palka suggested that if full use is made of the fact that $\partial D$ is a $k$-circle then an improved estimate [11, formula (17), p. 493] for these moduli might be true. They showed that if this were true then the better result that $f \in \operatorname{Lip}\left(\alpha_{1}, \bar{\Delta}\right)$, where

$$
\alpha_{1}=2(\arcsin k) / \pi
$$

would follow. Note that

$$
\alpha_{0} \sim\left(2 / \pi^{2}\right) k^{2} \quad \text { and } \quad \alpha_{1} \sim(2 / \pi) k \quad \text { as } k \rightarrow 0+.
$$

The following theorem shows that the conjecture that $f \in \operatorname{Lip}\left(\alpha_{1}, \bar{\Delta}\right)$ is false, at least for small $k$, and hence that the corresponding moduli conjecture is also false for such $k$. It seems possible that both conjectures are false for all $k<1$, though we are unable to prove this.

THEOREM 4. There is a number $k_{0}$ with $0<k_{0}<1$, such that for any $k \in\left(0, k_{0}\right)$ there exists a domain $D$ whose boundary $\Gamma$ is a k-circle passing through 0 and $\infty$ such that the following holds. If $g$ maps $H=\{z: \operatorname{Im} z>0\}$ conformally onto $D$ with $g(0)=0$, $g(\infty)=\infty$ and $|g(1)|=1$ then for all $z \in H$ with $|z|<1$ we have

$$
|g(z)| \geqslant A|z|^{\alpha}
$$

where $\alpha=B k^{\frac{3}{5}}$ and $A$ and $B$ are positive absolute constants.

In view of (3.5) and (3.6) we see that the conjecture that $g$ is Hölder continuous with exponent $\alpha_{1}$ is asymptotically false as $k \rightarrow 0+$. Perhaps some interest attaches to the question of finding the correct behaviour of $\alpha$ as $k \rightarrow 0+$, though this might be as difficult as finding the best possible $\alpha$ for each $k$.

We work in the upper half-plane $H$ rather than in $\Delta$ only for the sake of convenience. Also the fact that the domain $D$ of Theorem 4 is unbounded causes no 
difficulty. Pick a point $w_{0} \in \mathbb{C} \backslash \bar{D}$ and consider the domain $D_{1}$ onto which $H$ is mapped conformally by the function $g_{1}(z)=\left(g(z)-w_{0}\right)^{-1}$. It will be clear that the reasoning remains valid also for $g_{1}$ and $D_{1}$.

Theorem 4 yields the following corollary.

COROllary 2. For $0<k<k_{0}$, let $D$ be the domain of Theorem 4. Then there is a positive harmonic function $u$ in $D$, namely $u=\operatorname{Im} g^{-1}$, and a sequence $z_{n} \in D, z_{n} \rightarrow 0$, such that

$$
\limsup _{n \rightarrow \infty} \frac{u\left(z_{n}\right)}{d\left(z_{n}, \partial D\right)^{1 / \alpha}}<\infty
$$

where $\alpha=B k^{\frac{3}{2}}$ as before. In particular, (1.3) cannot hold for any exponent $\alpha>B k^{\frac{3}{2}}$.

Note that it is not claimed (and it is probably not true) that there exists a fixed absolute constant $B$ such that $\alpha=B k^{\frac{3}{2}}$ is exactly best possible in Theorem 4 and in Corollary 2. It is merely stated that if $B$ is suitably chosen and if $k$ is small enough, then (3.6) and (3.7) hold for the particular choice $\alpha=B k^{\frac{3}{8}}$ even though, for each fixed $k$, they might hold also for some slightly smaller values of $\alpha$.

REMARK. Lesley [10] defined the Jordan curve $\Gamma$ to be a $c$-quasiconformal curve if there are positive numbers $\delta$ and $c$ such that

$$
\left|z_{1}-z_{2}\right|+\left|z_{2}-z_{3}\right| \leqslant c\left|z_{1}-z_{3}\right|
$$

whenever $z_{1}, z_{2}, z_{3} \in \Gamma,\left|z_{1}-z_{3}\right| \leqslant \delta$ and $z_{2}$ is on that arc of $\Gamma$ determined by $z_{1}$ and $z_{3}$ that has the smaller Euclidean diameter. Let $f$ be a conformal mapping of $\Delta$ onto the interior of a bounded $c$-quasiconformal curve $\Gamma$. Lesley proved that then $f \in \operatorname{Lip}\left(\alpha_{2}, \bar{\Delta}\right)$ where $\alpha_{2}=(2 / \pi) \arcsin (1 / c)$ provided that $\Gamma$ is of bounded rotation [10, Theorem 4, p. 343], and that in general, $f \in \operatorname{Lip}\left(\alpha_{3}, \bar{\Delta}\right)$ where

$$
\alpha_{3}=(2 / \pi)[\arcsin (1 / c)]^{2}(\pi-\arcsin (1 / c))^{-1} \sim 2 \pi^{-2} c^{-2}
$$

as $c \rightarrow \infty$ [10, Theorem 2, p. 342]. He also gave an example [10, p. 351] where $\Gamma$ is not of bounded rotation and $f \notin \operatorname{Lip}(\alpha, \bar{\Delta})$ for any $\alpha>\alpha_{4}$, where $\alpha_{4} \sim 16 \pi^{-2} c^{-2}$ as $c \rightarrow \infty$.

Thus, if instead of characterizing a quasicircle globally by using the condition (3.1) that involves four points, we use locally the inequality (3.8) involving three points, then the correct order of magnitude of the best exponent of Hölder continuity is $c^{-2}$ as $c \rightarrow \infty$.

\section{An example}

4.1. The proof of Theorem 4 is established using the following example. For $k \leqslant k_{0}$, where $k_{0} \in(0,1)$ is to be chosen later, and for suitable positive constants $A_{1}, A_{2}, \ldots, A_{5}$ we define $r_{n}=\exp \left(A_{1} n k\right)$ for $-\infty<n<\infty$ and set

$$
\begin{aligned}
I_{4 n} & =\left[r_{2 n}, r_{2 n+1}\right], \\
I_{4 n+1} & =\left\{r e^{i \theta}: r=r_{2 n+1}, 0 \leqslant \theta \leqslant A_{2} k^{\frac{1}{2}}\right\}, \\
I_{4 n+2} & =\left\{r e^{i \theta}: r_{2 n+1} \leqslant r \leqslant r_{2 n+2}, \theta=A_{2} k^{\frac{1}{2}}\right\}, \\
I_{4 n+3} & =\left\{r e^{i \theta}: r=r_{2 n+2}, 0 \leqslant \theta \leqslant A_{2} k^{\frac{1}{2}}\right\},
\end{aligned}
$$




$$
\begin{aligned}
J_{4 n} & =\left\{r e^{i \theta}: r_{2 n} \exp \left(A_{3} k\right) \leqslant r \leqslant r_{2 n+1} \exp \left(-A_{3} k\right), \theta=A_{4} k\right\}, \\
J_{4 n+1} & =\left\{r e^{i \theta}: r=r_{2 n+1} \exp \left(-A_{3} k\right), A_{4} k \leqslant \theta \leqslant A_{2} k^{\frac{1}{2}}+A_{5} k\right\}, \\
J_{4 n+2} & =\left\{r e^{i \theta}: r_{2 n+1} \exp \left(-A_{3} k\right) \leqslant r \leqslant r_{2 n+2} \exp \left(A_{3} k\right), \theta=A_{2} k^{\frac{1}{2}}+A_{5} k\right\}, \\
J_{4 n+3} & =\left\{r e^{i \theta}: r=r_{2 n+2} \exp \left(A_{3} k\right), A_{4} k \leqslant \theta \leqslant A_{2} k^{\frac{1}{2}}+A_{5} k\right\} .
\end{aligned}
$$

If $k$ is small enough, depending on $A_{1}, \ldots, A_{5}$, and if $A_{1} / A_{3}$ is large enough, then $\gamma_{1}=\bigcup_{n--x}^{x} I_{n}$ and $\gamma_{2}=\bigcup_{n--x}^{x} J_{n}$ are disjoint open Jordan arcs from 0 to $\infty$ so that $\Gamma=\{0, \infty\} \cup \gamma_{1} \cup \gamma_{2}$ is a Jordan curve dividing the plane into two disjoint domains. For our domain $D$ we take that component of $\mathbb{C} \backslash \Gamma$ not containing the intervals $\left(r_{2 n+1}, r_{2 n+2}\right)$ for $-\infty<n<\infty$.

ThEOREM 5. For suitable constants $k_{0}, A_{1}, \ldots, A_{5}$ the curve $\Gamma$ is a $k$-circle for $0<k<k_{0}$.

4.2. The proof of Theorem 5 consists of a rather complicated verification of (3.1) and is given in Sections 6,7 and 8. Thus, assuming Theorem 5, we let $g$ be the mapping of $H$ onto $D$ as in Theorem 4 . Then $g$ has a $K$-quasiconformal extension to $\overline{\mathbb{C}}$, also denoted by $g$. Here $K$ depends on $k$ only. Thus it follows from [9, Theorem 9.1, p. 106], that

$$
L(r) \leqslant c_{2} l(r) \text { for all } r>0
$$

where $c_{2}$ is a constant depending on $K$ and hence on $k$ only while

$$
\begin{aligned}
L(r) & =\max \{|g(z)|:|z|=r\}, \\
l(r) & =\min \{|g(z)|:|z|=r\} .
\end{aligned}
$$

For a fixed $r$ with $0<r<1$, let $P_{r}$ be the family of paths lying in the semiannulus $H \cap\{z: r<|z|<1\}$ and joining the segments $(-1,-r)$ and $(r, 1)$. The modulus $M\left(P_{r}\right)$ of the family $P_{r}$ is given by $M\left(P_{r}\right)=\pi^{-1} \log (1 / r)$ (see, for example, [11, formula (21), p. 494]). Since $g$ is conformal in $H$ we have $M\left(P_{r}\right)=M\left(g\left(P_{r}\right)\right)$. Since $g(0)=0$ we may choose $r$ so small that $L(r)<l(1)$.

We seek a lower bound for $M\left(g\left(P_{r}\right)\right)$. More precisely, if $\rho$ is any non-negative Borel function in the plane such that

$$
\int_{\gamma} \rho d s \geqslant 1 \text { for all } \gamma \in g\left(P_{r}\right)
$$

then we seek a lower bound for $\iint_{\mathcal{C}} \rho^{2} d x d y$ in terms of $L(r)$. Note that $\gamma_{1}=g((0, \infty))$ and $\gamma_{2}=g((-\infty, 0))$ and that the set $D \cap\{z: L(r)<|z|<l(1)\}$ is covered by the paths in $g\left(P_{r}\right)$.

4.3. We choose integers $i$ and $j$ so that $r_{2 i-2}<L(r) \leqslant r_{2 i}, r_{2 j+1} \leqslant l(1)<r_{2 j+3}$. Then $i \leqslant j$ since $L(r)<l(1)$. The set

$$
\bigcup_{n=i}^{j}\left\{r e^{i \theta}: r_{2 n}<r<r_{2 n} \exp \left(A_{3} k\right), A_{4} k \leqslant \theta \leqslant A_{2} k^{\frac{1}{2}}\right\}=\bigcup_{n=i}^{j} S_{n},
$$

say, is contained in $D$. Consider a typical radial segment

$$
\gamma_{\theta}=\left\{r e^{i \theta}: r_{2 n}<r<r_{2 n} \exp \left(A_{3} k\right)\right\},
$$


where $A_{4} k \leqslant \theta \leqslant A_{2} k^{\frac{1}{2}}$ and $i \leqslant n \leqslant j$. Now $\gamma_{\theta}$ joins $\gamma_{1}$ to $\gamma_{2}$ so that $\gamma_{\theta}$ is a path of the family $g\left(P_{r}\right)$. If the function $\rho$ satisfies (4.2) we obtain by the Cauchy-Schwarz inequality that

$$
\begin{aligned}
\iint_{S_{n}} \rho^{2} d x d y & =\int_{A_{4} k}^{A_{k} k^{\frac{1}{2}}}\left\{\int_{r_{2 n}}^{r_{2 n} \exp \left(A_{3} k\right)}(\rho r)^{2} \frac{d r}{r}\right\} d \theta \\
& \geqslant \int_{A_{4} k}^{A_{2} k}\left\{\int_{r_{2 n} \frac{1}{2}}^{r_{2 n} \exp \left(A_{3} k\right)}(\rho r) \frac{d r}{r}\right\}\left\{\int_{r_{2 n}}^{r_{2 n} \exp \left(A_{3} k\right)} \frac{d r}{r}\right\}^{-1} d \theta \\
& \geqslant \frac{A_{2} k^{\frac{1}{2}}-A_{4} k}{A_{3} k}>\frac{A_{2}}{2 A_{3}} k^{-\frac{1}{2}}
\end{aligned}
$$

if $k<\left(2 A_{2} / A_{4}\right)^{2}$. Summing over $n$ we see that

$$
\iint_{\mathbb{C}} \rho^{2} d x d y \geqslant \frac{A_{2}}{2 A_{3}} k^{-\frac{1}{2}}(j-i+1) .
$$

From the definition of $i$ and $j$ we obtain

$$
\exp \left\{A_{1} k[5+2(\cdot j-i)]\right\}=\frac{r_{2 j+3}}{r_{2 i-2}} \geqslant \frac{l(1)}{L(r)} \geqslant \frac{1}{c_{2} L(r)}
$$

since, by $(4.1), l(1) \geqslant c_{2}^{-1} L(1) \geqslant c_{2}^{-1}$. It is here that we use the assumption that $|g(1)|=1$. Combining this with the known value of $M\left(g\left(P_{r}\right)\right)$ we obtain

$$
\begin{aligned}
\frac{1}{\pi} \log \frac{1}{r} & =M\left(g\left(P_{r}\right)\right) \geqslant \frac{A_{2}}{2 A_{3}} k^{-\frac{1}{2}}(j-i+1) \\
& \geqslant \frac{A_{2}}{4 A_{3}} k^{-\frac{1}{2}}\left\{-3+\frac{1}{A_{1} k} \log \frac{1}{c_{2} L(r)}\right\} .
\end{aligned}
$$

This may be reformulated as

$$
|g(z)| \geqslant c_{2}^{-1} L(r) \geqslant c_{2}^{-2} \exp \left(-3 A_{1} k\right)|z|^{\mid 3 k^{\frac{3}{2}}}
$$

where $B=4 A_{1} A_{3}\left(\pi A_{2}\right)^{-1}$. Thus (3.5) holds with $A=c_{2}^{-2} \exp \left(-3 A_{1} k\right)$ provided that $L(r)<l(1)$ (which implies that $i \leqslant j$ ). In fact, however, (4.3) is valid for all $z \in H$ with $|z|<1$. For if $|z|=r<1$ and $L(r) \geqslant l(1)$ then already from (4.1) we have

$$
|g(z)| \geqslant c_{2}^{-1} L(r) \geqslant c_{2}^{-1} l(1) \geqslant c_{2}^{-2}|z|^{s k^{\frac{3}{2}}}
$$

which is stronger than (4.3). Thus Theorem 4 is proved assuming Theorem 5. We note that nothing in the above part of the argument has restricted our choice of $A_{1}, \ldots, A_{5}$ and that the only restriction placed on $k$ so far is the condition that $k<\left(2 A_{2} / A_{4}\right)^{2}$.

The proof of Corollary 2 will be given in Section 9.

\section{Remarks on the example}

For the example $D$ of Section 4, the order of magnitude $k^{\frac{3}{2}}$ of the exponent of Hölder continuity of the function $g$ is sharp. To prove this, it suffices to find a good upper bound for $M\left(g\left(P_{r}\right)\right)$. We set

$$
\rho(z)= \begin{cases}(2|z| \arcsin k)^{-1}, & z \in D, l(r) \leqslant|z| \leqslant L(1), \\ 0, & \text { otherwise. }\end{cases}
$$


By [11, Lemma 3, p. 490], we have

and hence

$$
\int_{\gamma} \rho d s \geqslant i \text { for all } \gamma \in g\left(P_{r}\right)
$$

$$
M\left(g\left(P_{r}\right)\right) \leqslant \iint_{C} \rho^{2}(x+i y) d x d y=(2 \arcsin k)^{-2} \int_{l(r)}^{L(1)} \frac{d r}{r}\left\{\int_{r e^{i \theta} \in D} d \theta\right\}
$$

An obvious estimate shows that

$$
\int_{r e^{i \theta} \in D} d \theta \leqslant \max \left(A_{4} k, A_{5} k\right)+\psi(r),
$$

where $\psi(r)=A_{2} k^{\frac{1}{2}}$ if $r_{2 n} \leqslant r \leqslant r_{2 n} \exp \left(A_{3} k\right)$ or $r_{2 n+1} \exp \left(-A_{3} k\right) \leqslant r \leqslant r_{2 n+1}$, and $\psi(r)=0$ otherwise. With $A_{6}=\max \left(A_{4}, A_{5}\right)$ we obtain, since $\arcsin k \geqslant k$, that

$$
\frac{1}{\pi} \log \frac{1}{r}=M\left(g\left(P_{r}\right)\right) \leqslant \frac{1}{4 k^{2}}\left\{A_{6} k \log \frac{L(1)}{l(r)}+A_{2} k^{\frac{1}{2}} A_{3} k(q-p+1)\right\} .
$$

Here the integers $p$ and $q$ are defined by

$$
r_{p} \leqslant l(r)<r_{p+1}, \quad r_{q-1} \leqslant L(1)<r_{q},
$$

and hence

$$
\exp \left\{A_{1} k(q-p-2)\right\}=\frac{r_{q-1}}{r_{p+1}} \leqslant \frac{L(1)}{l(r)} .
$$

Combining this with (5.1) we see that

$$
\log \frac{1}{r} \leqslant \frac{\pi k}{4 k^{2}}\left\{\log \frac{L(1)}{l(r)}\right\}\left\{A_{6}+\frac{A_{2} A_{3} k^{\frac{1}{2}}}{A_{1} k}\right\}+\frac{3 \pi A_{2} A_{3}}{4 k^{\frac{1}{2}}} .
$$

Unwinding all this we get for $|z|=r$ that

$$
|g(z)| \leqslant c_{2} l(r) \leqslant B_{1}|z|^{B_{2} k^{\frac{3}{2}}}
$$

for suitable constants $B_{1}$ and $B_{2}$.

It follows that to decrease the order of magnitude $k^{\frac{3}{2}}$ of the exponent of Hölder continuity, if possible at all, one would have to consider a $k$-circle that in some essential way differs from $\partial D$ for the above domain $D$.

\section{Proof of Theorem 5. Case I}

To prove Theorem 5 we set $Z=\left\{z_{1}, z_{2}, z_{3}, z_{4}\right\}$ where the $z_{i}$ belong to $\gamma_{1} \cup \gamma_{2}$ of Section 4. We thus omit the cases when $z_{i}=0, \infty$ for some $i$, but (3.1) will follow in these cases by continuity. Without loss of generality we suppose that $Z$ is positively oriented and set

$$
S(Z)=k\left\{\left|z_{1}-z_{2}\right|\left|z_{3}-z_{4}\right|+\left|z_{1}-z_{4}\right|\left|z_{2}-z_{3}\right|\right\} .
$$

We put $z_{j}=\rho_{j} \exp \left(i \theta_{j}\right)$, where $0<\rho_{j}<\infty$ and $0 \leqslant \theta_{j}<\pi / 2$, for $1 \leqslant j \leqslant 4$. This already imposes a restriction of the form $k \leqslant k_{0}$ as it assumes that

$$
\max \left(A_{4} k, A_{2} k^{\frac{1}{2}}+A_{5} k\right)<\frac{1}{2} \pi \text {. }
$$


There are five cases to consider.

Case I. All the $z_{j}, 1 \leqslant j \leqslant 4$, lie on $\gamma_{1}$.

Case II. All the $z_{j}$ lie on $\gamma_{2}$.

Case III. $z_{1} \in \gamma_{2}$ but $z_{2}, z_{3}, z_{4}$ lie on $\gamma_{1}$.

Case IV. $z_{1}, z_{2}, z_{3}$ lie on $\gamma_{2}$ but $z_{4} \in \gamma_{1}$.

Case V. $z_{1}, z_{2}$ lie on $\gamma_{2}$ and $z_{3}, z_{4}$ lie on $\gamma_{1}$.

Case II is similar to Case I and Case IV to Case III; so we shall consider only Cases I, III and V.

Case I. Because of the orientation of $Z$, we have $\rho_{1} \leqslant \rho_{2} \leqslant \rho_{3} \leqslant \rho_{4}$. We consider several subcases of Case I, assuming from the start that $0<k \leqslant k_{0}<\frac{1}{32}$. We shall denote by $C$ a positive absolute constant, not necessarily the same at every occurrence.

Case I(a). All the $z_{i}$ belong to $I_{v} \cup I_{v+1}$ for some fixed $v$.

If all the points $z_{i}$ belong to $I_{v}$ for some fixed $v$ then, since $I_{v}$ is contained in a line or a circle, (3.1) holds with $k=1$. Suppose that $Z \subset I_{v} \cup I_{v+1}$, where $I_{v}=\left[r_{n}, r_{n+1}\right]$ and $I_{v+1}=\left\{r e^{i \theta}: r=r_{n+1}, 0 \leqslant \theta \leqslant A_{2} k^{\frac{1}{2}}<\frac{1}{2} \pi\right\}$, for some $n$. The Möbius transformation $T_{n}(z)=\left(r_{n+1}+z\right)\left(r_{n+1}-z\right)^{-1}$ maps $I_{v} \cup I_{v+1}$ onto a subset of $\mathbb{R}^{+} \cup i \mathbb{R}^{+}$. Now the boundary of the first quadrant is a $k_{1}$-circle for $k_{1}=\sin \frac{1}{4} \pi=2^{-\frac{1}{2}}$ (cf. (3.2)). Hence (3.1) will hold whenever $k \leqslant 2^{-\frac{1}{2}}$. The other possibilities for $I_{v} \cup I_{v+1}$ are dealt with analogously.

From now on we assume that there is no $v$ such that $Z \subset I_{v} \cup I_{v+1}$.

Case I(b). $\left|z_{2}-z_{4}\right| \leqslant \frac{1}{2} A_{1} k\left|z_{4}\right|<\left|z_{4}\right|\left(1-\exp \left(-A_{1} k\right)\right)$.

We assume that $A_{1} k<A_{2} k^{\frac{1}{2}}$, which is true if $k_{0}$ is small enough. Then

$$
\left|z_{2}-z_{3}\right|+\left|z_{3}-z_{4}\right| \leqslant C\left|z_{2}-z_{4}\right|,
$$

and (3.1) will hold provided that $C k \max \left(\left|z_{1}-z_{2}\right|,\left|z_{1}-z_{4}\right|\right) \leqslant\left|z_{1}-z_{3}\right|$.

By the geometry of $\gamma_{1}$ and by our assumption, we have $\left|z_{1}-z_{2}\right| \leqslant C\left|z_{1}-z_{3}\right|$. Next, if $z_{2} \in I_{v}$, then our assumption implies that $z_{3}, z_{4} \in I_{v} \cup I_{v+1}$. Therefore, by our assumption, we have $z_{1} \notin I_{v}$, and further $z_{1} \notin I_{v-1} \cup I_{v}$ if it happens that $z_{3}, z_{4} \in I_{v}$. Now geometric considerations show that $\left|z_{3}-z_{4}\right| \leqslant C\left|z_{1}-z_{3}\right|$. Using this together with the inequality $\left|z_{1}-z_{4}\right| \leqslant\left|z_{1}-z_{3}\right|+\left|z_{3}-z_{4}\right|$ we see that (3.1) holds for all sufficiently small $k$.

Case I(c). $\quad\left|z_{1}-z_{3}\right| \leqslant \frac{1}{2} A_{1} k\left|z_{3}\right|$.

This is analogous to Case $\mathrm{I}(\mathrm{b})$, and we omit the details.

It is elementary to show that if $\left|w_{1}\right| \leqslant\left|w_{2}\right| \leqslant\left|w_{3}\right|$ and $0 \leqslant \arg w_{j} \leqslant A_{2} k^{\frac{1}{2}}<\frac{1}{2} \pi$ for $1 \leqslant j \leqslant 3$ then

$$
\begin{aligned}
\left|w_{1}-w_{2}\right|+\left|w_{2}-w_{3}\right| & \leqslant\left|w_{3}\right|-\left|w_{1}\right|+2 A_{2} k^{\frac{1}{2}}\left|w_{2}\right| \\
& \leqslant\left|w_{3}-w_{1}\right|+2 A_{2} k^{\frac{1}{2}}\left|w_{2}\right| .
\end{aligned}
$$

Several estimates below will be based on this.

From now on, we may assume that

$$
\left|z_{1}-z_{3}\right| \geqslant \frac{1}{2} A_{1} k\left|z_{3}\right| \text { and }\left|z_{2}-z_{4}\right| \geqslant \frac{1}{2} A_{1} k\left|z_{4}\right| .
$$


Case I(d). We have

$$
\left|z_{1}-z_{4}\right| \leqslant\left|z_{1}-z_{2}\right|+\left|z_{2}-z_{3}\right|+\left|z_{3}-z_{4}\right|
$$

so that

$$
\begin{aligned}
k^{-1} S(Z) & =\left|z_{1}-z_{2}\right|\left|z_{3}-z_{4}\right|+\left|z_{2}-z_{3}\right|\left|z_{1}-z_{4}\right| \\
& \leqslant\left(\left|z_{1}-z_{2}\right|+\left|z_{2}-z_{3}\right|\right)\left(\left|z_{2}-z_{3}\right|+\left|z_{3}-z_{4}\right|\right) \\
& \leqslant\left(\left|z_{1}-z_{3}\right|+2 A_{2} k^{\frac{1}{2}}\left|z_{2}\right|\right)\left(\left|z_{2}-z_{4}\right|+2 A_{2} k^{\frac{1}{2}}\left|z_{3}\right|\right)
\end{aligned}
$$

by (6.1). Hence (3.1) holds provided that

$$
\left|z_{1}-z_{3}\right|+2 A_{2} k^{\frac{1}{2}}\left|z_{2}\right| \leqslant k^{-\frac{1}{2}}\left|z_{1}-z_{3}\right|
$$

and

$$
\left|z_{2}-z_{4}\right|+2 A_{2} k^{\frac{1}{2}}\left|z_{3}\right| \leqslant k^{-\frac{1}{2}}\left|z_{2}-z_{4}\right| .
$$

Clearly (6.3) holds whenever

$$
\left|z_{1}-z_{3}\right| \geqslant 4 A_{2} k\left|z_{2}\right|
$$

if $k \leqslant \frac{1}{4}$ as we assume. So if we choose $A_{1}$ to be greater than $8 A_{2}$ then (6.3) is a consequence of our assumption (6.2). Similarly, (6.4) follows from (6.2).

This completes our treatment of Case I.

\section{Case III}

It is easily verified that the distance $d\left(z, \gamma_{2}\right)$ from $z \in \gamma_{1}$ to $\gamma_{2}$ satisfies

$$
d\left(z, \gamma_{2}\right) \geqslant C k|z| \min \left(A_{3}, A_{4}, A_{5}\right)
$$

for a suitable positive constant $C$ and we write, similarly,

$$
d\left(z, \gamma_{1}\right) \geqslant A_{7} k|z| \text { for } z \in \gamma_{2}
$$

where $A_{7}$ is large if each of $A_{3}, A_{4}, A_{5}$ is chosen to be large enough. Because of the orientation of $Z$, we have $\rho_{2} \leqslant \rho_{3} \leqslant \rho_{4}$.

So if $z_{1} \in \gamma_{2}$ and $z_{3} \in \gamma_{1}$ then

$$
\left|z_{1}-z_{3}\right| \geqslant A_{7} k \max \left\{\left|z_{1}\right|,\left|z_{3}\right|\right\}
$$

Case III(a). $\rho_{1} \geqslant 2 \rho_{4}$.

Here we have the estimate

$$
S(Z) \leqslant k\left\{\left(2 \rho_{1}\right)\left(2 \rho_{4}\right)+\left(2 \rho_{1}\right)\left(2 \rho_{3}\right)\right\} \leqslant 8 k \rho_{1} \rho_{4},
$$

while $\left|z_{1}-z_{3}\right| \geqslant \rho_{1}-\rho_{3} \geqslant \rho_{1}-\rho_{4} \geqslant \rho_{1} / 2$. Thus (3.1) holds if $\left|z_{2}-z_{4}\right| \geqslant 16 k \rho_{4}$. But if $\left|z_{2}-z_{4}\right|<16 k \rho_{4}$ and $A_{1}$ is large enough, then geometric considerations show that $\left|z_{2}-z_{3}\right|+\left|z_{3}-z_{4}\right| \leqslant 3\left|z_{2}-z_{4}\right|$. Thus

$$
S(Z) \leqslant 6 k \rho_{1}\left|z_{2}-z_{4}\right| \leqslant 12 k\left|z_{2}-z_{4}\right|\left|z_{1}-z_{3}\right| .
$$

Hence (3.1) holds if $12 k \leqslant 1$.

Case III(b). $\quad \rho_{1}<2 \rho_{4}$ and $\left\{z_{2}, z_{3}, z_{4}\right\} \subset I_{v} \cup I_{v+1}$ for some $v$.

In this case $\left|z_{2}-z_{3}\right|+\left|z_{3}-z_{4}\right| \leqslant 3\left|z_{2}-z_{4}\right|$, say, from the geometry of $\gamma_{1}$, so that

$$
S(Z) \leqslant 3 k\left|z_{2}-z_{4}\right| \max \left\{\left|z_{1}-z_{2}\right|,\left|z_{1}-z_{4}\right|\right\} \text {. }
$$


We have $\left|z_{1}-z_{3}\right| \geqslant A_{7} k\left|z_{3}\right| \geqslant A_{7} k \rho_{4} / 2$ by (7.1) and since $z_{3}, z_{4} \in I_{v} \cup I_{v+1}$. Further, $\left|z_{1}-z_{2}\right|$ and $\left|z_{1}-z_{4}\right|$ are less than $3 \rho_{4}$. Thus

$$
S(Z) \leqslant 9 k \rho_{4}\left|z_{2}-z_{4}\right| \leqslant\left|z_{1}-z_{3}\right|\left|z_{2}-z_{4}\right|
$$

if $A_{7}$ is large enough, and (3.1) holds.

Case III(c). $\rho_{1}<2 \rho_{4}$ but $\left\{z_{2}, z_{3}, z_{4}\right\} \notin I_{v} \cup I_{v+1}$ for any $v$.

The latter assumption implies that $\left|z_{2}-z_{4}\right| \geqslant A_{1} k\left|z_{4}\right| / 2$ so that (6.4) holds. If $\rho_{1} \leqslant \rho_{2}$ then (6.1) and (7.1) imply that (6.3) holds, so that we now obtain (3.1) as in Case I(d). If $\rho_{4} \leqslant \rho_{1}<2 \rho_{4}$ then we have

$$
\begin{aligned}
\left|z_{i}-z_{j}\right| & \leqslant\left|\rho_{i}-\rho_{j}\right|+\left|\theta_{i}-\theta_{j}\right| \min \left\{\rho_{i}, \rho_{j}\right\} \\
& \leqslant\left|\rho_{i}-\rho_{j}\right|+2 A_{2} k^{\frac{1}{2}} \min \left\{\rho_{i}, \rho_{j}\right\}
\end{aligned}
$$

for all $i, j$, which implies, after some calculations, that

$$
S(Z) \leqslant k\left(\rho_{1}-\rho_{3}\right)\left(\rho_{4}-\rho_{2}\right)+2 A_{2} k^{\frac{3}{2}} \rho_{2}\left(\rho_{1}-\rho_{4}\right)+4 A_{2}^{2} k^{2} \rho_{2} \rho_{4} \leqslant\left|z_{1}-z_{3}\right|\left|z_{2}-z_{4}\right|
$$

if $k$ is small enough. Note that (7.1) holds and that $\rho_{1}-\rho_{4} \leqslant \rho_{1}-\rho_{3} \leqslant\left|z_{1}-z_{3}\right|$.

If $\rho_{2} \leqslant \rho_{1} \leqslant \rho_{4}$ then (7.2) gives

$$
\begin{aligned}
S(Z) \leqslant & k\left\{\rho_{4}\left[\left(\rho_{1}-\rho_{2}\right)+\left(\rho_{3}-\rho_{2}\right)\right]-\rho_{3}\left(\rho_{1}-\rho_{2}\right)-\rho_{1}\left(\rho_{3}-\rho_{2}\right)\right\} \\
& +2 A_{2} k^{\frac{3}{2}}\left\{\rho_{2}\left[\left(\rho_{4}-\rho_{3}\right)+\left(\rho_{4}-\rho_{1}\right)\right]+\rho_{3}\left(\rho_{1}-\rho_{2}\right)+\rho_{1}\left(\rho_{3}-\rho_{2}\right)\right\} \\
& +4 A_{2}^{2} k^{2} \rho_{2} \max \left\{\rho_{1}, \rho_{3}\right\} \\
\leqslant & k \rho_{4}\left[\left(\rho_{1}-\rho_{2}\right)+\left(\rho_{3}-\rho_{2}\right)\right]+8 A_{2} k^{\frac{3}{2}}\left(\rho_{4}-\rho_{2}\right) \max \left\{\rho_{1}, \rho_{3}\right\} \\
& +4 A_{2}^{2} k^{2} \rho_{2} \max \left\{\rho_{1}, \rho_{3}\right\} .
\end{aligned}
$$

The sum of the last two terms does not exceed

$$
8\left(A_{2}^{2}+A_{2}\right) A_{7}^{-1}\left|z_{1}-z_{3}\right|\left|z_{2}-z_{4}\right| \leqslant \frac{1}{2}\left|z_{1}-z_{3}\right|\left|z_{2}-z_{4}\right|
$$

if $A_{1}>1$ and $A_{7}$ is large enough. If $2 \rho_{2} \leqslant \rho_{4}$ then $\left|z_{2}-z_{4}\right| \geqslant \rho_{4} / 2$ and

$$
k \rho_{4}\left[\rho_{1}-\rho_{2}+\rho_{3}-\rho_{2}\right] \leqslant 4 k\left|z_{2}-z_{4}\right| \max \left\{\rho_{1}, \rho_{3}\right\} \leqslant \frac{1}{2}\left|z_{1}-z_{3}\right|\left|z_{2}-z_{4}\right|
$$

by (7.1). If $2 \rho_{2}>\rho_{4}$ then $\rho_{1}+\rho_{3}-2 \rho_{2} \leqslant 2\left(\rho_{4}-\rho_{2}\right)$ while

$$
k \rho_{4} \leqslant 2 k \max \left\{\rho_{1}, \rho_{3}\right\} \leqslant 2 A_{7}^{-1}\left|z_{1}-z_{3}\right|
$$

so that (3.1) is valid. This completes our treatment of Case III.

\section{Case V}

From the orientation of $Z$ we see that $\rho_{1} \geqslant \rho_{2}$ and $\rho_{3} \leqslant \rho_{4}$.

Case V(a). $\quad \rho_{1} \geqslant 2 \rho_{4}$.

Here we have

$$
\begin{aligned}
S(Z) & \leqslant k\left\{\left(2 \rho_{1}\right)\left(2 \rho_{4}\right)+\left(\rho_{2}+\rho_{3}\right)\left(\rho_{1}+\rho_{4}\right)\right\} \\
& \leqslant k\left\{4 \rho_{1} \rho_{4}+\left(3 \rho_{1} / 2\right)\left(2 \max \left\{\rho_{2}, \rho_{4}\right\}\right)\right\} \leqslant 7 k \rho_{1} \max \left\{\rho_{2}, \rho_{4}\right\}
\end{aligned}
$$

since $\rho_{2}+\rho_{3} \leqslant \rho_{2}+\rho_{4} \leqslant 2 \max \left\{\rho_{2}, \rho_{4}\right\}$. On the other hand,

$$
\left|z_{1}-z_{3}\right| \geqslant \rho_{1}-\rho_{3} \geqslant \rho_{1}-\rho_{4} \geqslant \rho_{1} / 2 \text { and }\left|z_{2}-z_{4}\right| \geqslant A_{7} k \max \left\{\rho_{2}, \rho_{4}\right\} .
$$

Thus once again (3.1) holds if $A_{7}$ is chosen large enough. 
Case V(b). Either $\rho_{4} \geqslant 2 \rho_{1}$ or $2 \rho_{2} \leqslant \rho_{3}$ or $2 \rho_{3} \leqslant \rho_{2}$.

These are dealt with in the same way as Case V(a).

Case V(c). $\frac{1}{2} \leqslant \rho_{4} / \rho_{1} \leqslant 2$ and $\frac{1}{2} \leqslant \rho_{3} / \rho_{2} \leqslant 2$.

This is really the heart of the matter. If $\max \left\{\rho_{2}, \rho_{3}\right\} \leqslant \frac{1}{2} \min \left\{\rho_{1}, \rho_{4}\right\}$ then $\left|z_{1}-z_{3}\right|\left|z_{2}-z_{4}\right| \geqslant \rho_{1} \rho_{4} / 4$, while

$$
S(Z) \leqslant k\left\{\left(2 \rho_{1}\right)\left(2 \rho_{4}\right)+\left(2 \rho_{1}\right)\left(2 \rho_{4}\right)\right\}=8 k \rho_{1} \rho_{4}
$$

so that (3.1) holds if $k \leqslant \frac{1}{32}$. Henceforth we may assume that

so that

$$
\max \left\{\rho_{2}, \rho_{3}\right\}>\frac{1}{2} \min \left\{\rho_{1}, \rho_{4}\right\}
$$

$$
\max \left\{\rho_{1}, \rho_{2}, \rho_{3}, \rho_{4}\right\} \leqslant 8 \min \left\{\rho_{1}, \rho_{2}, \rho_{3}, \rho_{4}\right\}=8 R,
$$

say. We assume further that $\rho_{2} \leqslant \rho_{3}$ and hence

$$
R=\rho_{2} \leqslant \rho_{3} \leqslant \rho_{4} \leqslant 8 R, \quad \rho_{2} \leqslant \rho_{1} \leqslant 2 \rho_{4}, \quad \rho_{1} \leqslant 8 R .
$$

The situation is entirely analogous when $\rho_{3}<\rho_{2}$. Now by (6.1) and the relation between $\gamma_{1}$ and $\gamma_{2}$,

$$
\left|z_{2}-z_{3}\right|+\left|z_{3}-z_{4}\right| \leqslant\left|z_{2}-z_{4}\right|+C A_{2} k^{\frac{1}{2}} R
$$

for some positive absolute constant $C$. If $\left|z_{2}-z_{4}\right| \geqslant A_{2} k^{\frac{1}{2}} R$ then

$$
\begin{aligned}
S(Z) & \leqslant k(1+C)\left|z_{2}-z_{4}\right| \max \left\{\left|z_{1}-z_{2}\right|,\left|z_{1}-z_{4}\right|\right\} \\
& \leqslant 16 k(1+C) R\left|z_{2}-z_{4}\right| \leqslant\left|z_{1}-z_{3}\right|\left|z_{2}-z_{4}\right|
\end{aligned}
$$

by (7.1) if $A_{7}$ is large enough.

Suppose now that $\left|z_{2}-z_{4}\right|<A_{2} k^{\frac{1}{2}} R$ and that $\rho_{1} \geqslant \rho_{4}+A_{2} k^{\frac{1}{2}} R$. Then

$$
\left|z_{1}-z_{3}\right| \geqslant \rho_{1}-\rho_{3} \geqslant \rho_{1}-\rho_{4} \geqslant A_{2} k^{\frac{1}{2}} R
$$

and $\left|z_{2}-z_{4}\right| \geqslant A_{j} k R$ so that

$$
\left|z_{1}-z_{3}\right|\left|z_{2}-z_{4}\right| \geqslant A_{2} A_{7} k^{\frac{3}{2}} R^{2} .
$$

Also

$$
S(Z) \leqslant 2 \rho_{1} k\left(\left|z_{3}-z_{4}\right|+\left|z_{2}-z_{3}\right|\right) \leqslant 16 R k\left(\left|z_{2}-z_{3}\right|+\left|z_{3}-z_{4}\right|\right) .
$$

To obtain (3.1) we are required to prove that

$$
\left|z_{2}-z_{3}\right|+\left|z_{3}-z_{4}\right| \leqslant\left(A_{7} / 16\right) A_{2} k^{\frac{1}{2}} R .
$$

Consideration of where $z_{3}$ can lie on $\gamma_{1}$ in view of the restrictions $\rho_{2} \leqslant \rho_{3} \leqslant \rho_{4}$ and $\left|z_{2}-z_{4}\right| \leqslant A_{2} k^{\frac{1}{2}} R$, shows that (8.1) will hold if $A_{7}$ is larger than some absolute constant.

Suppose next that $\left|z_{2}-z_{4}\right| \leqslant A_{2} k^{\frac{1}{2}} R$ and that $\rho_{1} \leqslant \rho_{4}+A_{2} k^{\frac{1}{2}} R$. Then by considering where $z_{1}$ and $z_{3}$ can possibly lie in view of all the assumptions we see that all the quantities $\left|z_{1}-z_{2}\right|,\left|z_{3}-z_{4}\right|,\left|z_{2}-z_{3}\right|$, and hence also $\left|z_{1}-z_{4}\right|$, are less than $C_{1} A_{2} k^{\frac{1}{2}} R$ for some suitable constant $C_{1}$. To see this for $\left|z_{1}-z_{2}\right|$, note that also $\rho_{2} \leqslant \rho_{1}$. Thus $S(Z) \leqslant 2 C_{1}^{2} A_{2}^{2} k^{2} R^{2}$ and $\left|z_{1}-z_{3}\right| \geqslant A, k R$. Hence (3.1) holds if

$$
\left|z_{2}-z_{4}\right| \geqslant 2 C_{1}^{2} A_{2}^{2} A_{7}^{-1} k R \text {. }
$$

Having chosen $A_{3}, A_{4}, A_{5}$ so large that $A_{7}$ is large enough we now choose $A_{1}$ so large that, in addition to any previous requirements,

$$
\exp \left(A_{1} k / 2\right) \geqslant 1+2 C_{1}^{2} A_{2}^{2} A_{7}^{-1} k \text { for } 0<k \leqslant 1 .
$$


Then, if (8.2) fails to hold, we have

$$
\rho_{4} \leqslant \rho_{2}\left(1+2 C_{1}^{2} A_{2}^{2} A_{7}^{-1} k\right) \leqslant \rho_{2} \exp \left(A_{1} k / 2\right) \leqslant \rho_{3} \exp \left(A_{1} k / 2\right),
$$

since $R \leqslant \rho_{2} \leqslant \rho_{3}$. Considering how $z_{2}, z_{3}, z_{4}$ can be located we see that, for a suitable constant $C_{2}>0$,

$$
\begin{aligned}
\left|z_{2}-z_{3}\right|+\left|z_{3}-z_{4}\right| & \leqslant\left|z_{2}-z_{4}\right|+C_{2} k R \max \left\{A_{4}, A_{5}\right\} \\
& \leqslant\left|z_{2}-z_{4}\right|\left(1+C_{2} A_{7}^{-1} \max \left\{A_{4}, A_{5}\right\}\right),
\end{aligned}
$$

since, in any case, $\left|z_{2}-z_{4}\right| \geqslant A_{7} k R$. Thus (3.1) is satisfied if

$$
k \max \left\{\left|z_{1}-z_{2}\right|,\left|z_{1}-z_{4}\right|\right\}\left(1+C_{2} A_{7}^{-1} \max \left\{A_{4}, A_{5}\right\}\right) \leqslant\left|z_{1}-z_{3}\right| .
$$

But both $\left|z_{1}-z_{2}\right|$ and $\left|z_{1}-z_{4}\right|$ are less than $2 \rho_{4} \leqslant 16 R$ while $\left|z_{1}-z_{3}\right| \geqslant A_{7} k R$. Thus (8.3) is valid and hence (3.1) is fulfilled provided that $A_{7}$ is large enough.

This completes our treatment of Case V. Theorem 5 is thus proved for all configurations of $Z$.

\section{Proof of Corollary 2}

Let the assumptions of Corollary 2 be satisfied. Since

$$
\left(\operatorname{Im} g^{-1}(z)\right)^{\alpha} \leqslant\left|g^{-1}(z)\right|^{\alpha} \leqslant A^{-1}|z|
$$

as $z \rightarrow 0$ in $D$ by (3.6), it suffices to find a sequence $w_{n} \in D, w_{n} \rightarrow 0$ such that

$$
\left|w_{n}\right| \leqslant A_{1} d\left(w_{n}, \partial D\right)
$$

for all $n$ and for some constant $A_{1}$. Let the arcs of $\partial D$ joining 0 to $\infty$ be $\gamma_{1}$ and $\gamma_{2}$ as before. For $r>0$, we find an open arc $\gamma$ of $\{z:|z|=r\}$ contained in $D$ with endpoints $\zeta_{1}$ and $\zeta_{2}$ on $\gamma_{1}$ and $\gamma_{2}$, respectively. For $i=1,2$, we set $d_{i}(w)=d\left(w, \gamma_{i}\right)$. As $w$ moves from $\zeta_{1}$ to $\zeta_{2}$ along $\gamma$, the function $d_{1}(w) / d_{2}(w)$ changes continuously from 0 to $\infty$. Thus there is $w_{0} \in \gamma$ such that $d_{1}\left(w_{0}\right)=d_{2}\left(w_{0}\right)=d\left(w_{0}, \partial D\right)=d$, say. We choose $b_{i} \in \gamma_{i}$ with $\left|b_{i}-w_{0}\right|=d$, for $i=1,2$.

We wish to prove (9.1) with $w_{n}$ replaced by $w_{0}$. If $b_{1}=0$ or $b_{2}=0$ then $d=\left|w_{0}\right|$ and (9.1) holds. Otherwise $b_{1} \neq b_{2}$, and since $\partial D$ is a quasicircle, there is a constant $c_{3}$ depending on $k$ only such that

$$
M=\max \left\{\left|b_{1}\right|,\left|b_{2}\right|\right\} \leqslant \operatorname{diam} J \leqslant c_{3}\left|b_{1}-b_{2}\right|,
$$

where $J$ is the arc of $\partial D$ joining $b_{1}$ to $b_{2}$ and going through the origin. Hence $M \leqslant c_{3}\left\{\left|b_{1}-w_{0}\right|+\left|b_{2}-w_{0}\right|\right\}=2 c_{3} d$.

If $M \geqslant\left|w_{0}\right| / 2$ then (9.1) holds. Otherwise, neither $\gamma_{1}$ nor $\gamma_{2}$ contains any points in $\left\{w:\left|w-w_{0}\right|<\left|w_{0}\right| / 2\right\}$, and so $d \geqslant\left|w_{0}\right| / 2$. Thus in any case (9.1) holds, and Corollary 2 is proved.

\section{References}

1. L. V. Ahlfors, 'Quasiconformal reflections', Acta Math. 109 (1963) 291-301.

2. J. M. Anderson, F. W. Gehring and A. Hinkkanen, 'Polynomial approximation in quasidisks', Differential geometry and complex analysis, H. E. Rauch Memorial Volume (Springer, Berlin, 1985) 75-86.

3. D. K. Blevins, 'Some properties of domains bounded by quasiconformal circles', Ph.D. Thesis, University of Michigan 1972.

4. D. K. Blevins, 'Harmonic measure and domains bounded by quasiconformal circles', Proc. Amer. Math. Soc. 41 (1973) 559-564.

5. P. L. Duren, Theory of $H^{p}$ spaces (Academic Press, New York, 1970). 
6. G. H. HARDY and J. E. LiTrLeWOOd, 'Some properties of fractional integrals II', Math. Z. 34 (1932) 403-439.

7. Ü. KURAN, 'On positive superharmonic functions in a-admissible domains', J. London Math. Soc. (2) 29 (1984) 269-275.

8. Ü. KURAN and J. L. SCHIFF, 'A uniqueness theorem for nonnegative superharmonic functions in plane domains', J. Math. Anal. Appl. 93 (1983) 195-205.

9. O. LeHTO and K. I. VIRTANEN, Quasiconformal mappings in the plane (Springer, Berlin, 1973).

10. F. D. LeSLEY, 'Hölder continuity of conformal mappings at the boundary via the strip method', Indiana Univ. Math. J. 31 (1982) 341-354.

11. R. NÄKKı and B. PalKa, 'Quasiconformal circles and Lipschitz classes', Comment. Math. Helv. 55 (1980) 485-498.

12. Сн. Pommerenke, Univalent functions (Vandenhoeck \& Ruprecht, Göttingen, 1975).

\author{
Department of Mathematics \\ University College \\ Gower Street \\ London WC1E 6BT
}

\author{
Department of Mathematics \\ University of Michigan \\ Ann Arbor, Michigan 48109-1003 \\ USA
}

Current address:

Department of Mathematics

The University of Texas at Austin Austin, Texas 78712

USA 